\title{
Higher order curvature corrections to the field emission current density
}

\author{
Debabrata Biswas ${ }^{1,2, a)}$ and Rajasree Ramachandran ${ }^{1,2}$ \\ 1) Bhabha Atomic Research Centre, Mumbai 400 085, INDIA \\ ${ }^{2)}$ Homi Bhabha National Institute, Mumbai 400 094, INDIA
}

A simple expression for the Gamow factor is obtained using a second order curvature corrected tunneling potential. Our results show that it approximates accurately the 'exact-WKB' transmission coefficient obtained by numerically integrating over the tunneling region to obtain the Gamow factor. The average difference in current density using the respective transmission coefficients is about $1.5 \%$, across a range of work-functions $\phi \in[3-5.5] \mathrm{eV}$, Fermi energy $\mathcal{E}_{F} \in[5-10] \mathrm{eV}$, local electric fields $E_{l} \in[3-9] \mathrm{eV}$ and radius of curvature $R \geq 5 \mathrm{~nm}$. An easy-to-use correction factor $\lambda_{P}$ is also provided to approximately map the 'exact-WKB' current density to the 'exact' current density in terms of $\mathcal{E}_{F} / \phi$. The average error on using $\lambda_{P}$ is found to be around $3.5 \%$ This is a vast improvement over the average error of $15 \%$ when $\lambda_{P}=1$. Finally, an analytical expression for the curvature-corrected current density is obtained using the Gamow factor. It is found to compare well with the 'exact-WKB' current density even at small values of local electric field and radius of curvature.

\section{INTRODUCTION}

Field emission refers to the quantum mechanical tunneling of electrons from the surface of a conductor on application of an external electric field 1 12]. It requires local surface fields upwards of $3 \mathrm{~V} / \mathrm{nm}$ for a measurable current to flow. Such high fields can be easily achieved on the surface of sharp tips due to the convergence of field lines resulting in the enhancement of the local field $E_{l}$ over the asymptotic or macroscopic applied field, $E_{0}$ by a factor $\gamma=E_{l} / E_{0}$. In modern-day usage, $E_{l}$ embodies the essence of curved emitters and the field enhancement factor $\gamma$ is the focus of much research 13 .16.

In applying the field emission formalism ${ }^{144778}$ to curved emitters, it is implicitly assumed that the local field $E_{l}$ at any point on the surface remains constant along the outward normal till the end of the classically forbidden region. For $\phi=4.5 \mathrm{eV}$ and $E_{l}>4 \mathrm{~V} / \mathrm{nm}$, this is typically less that $1.5 \mathrm{~nm}$. The corresponding potential energy is thus expressed as $V_{\text {ext }}=-q E_{l} s$ where $s$ is the normal distance from the point on the surface of the emitter.

For a curved emitting tip with apex radius of curvature $R_{a}<100 \mathrm{~nm}$, the electric field can no longer be assumed to be constant in the tunneling region and does in fact fall off sharply resulting in a reduced tunneling current ${ }^{11}$. The current density formula must therefore reflect this dependence on $R_{a}$ accurately for it to be applied seamlessly in practical applications.

The first correction to the electrostatic potential at the apex of an axially symmetric emitter was provided in 2015. It was established under general considerations that along the symmetry axis $\frac{17}{}$,

$$
V_{\mathrm{ext}}(s)=-q E_{l} s\left[1-\frac{s}{R_{a}}+\mathcal{O}\left(\frac{s}{R_{a}}\right)^{2}\right]
$$

where $q$ is the electronic charge.

Using analytically solvable models such as the hemiellipsoid and hyperboloid diodes, it was subsequently 18 shown in 2018 that at points close to the apex, the potential has the form

$$
V_{\mathrm{ext}}(s) \approx-q E_{l} s\left[1-c_{1} \frac{s}{R_{2}}+c_{2} \frac{4}{3}\left(\frac{s}{R_{2}}\right)^{2}+\mathcal{O}\left(s^{3}\right)\right]
$$

where $R_{2}$ is the second principal radius of curvature at any point on the surface of the emitter while at the apex, $R_{2}=R_{a}$. The quantities $c_{1}$ and $c_{2}$ depend on the point on the emitter surface. For points close to the apex $c_{1} \approx$ $1 \approx c_{2}$.

Recently $\sqrt{19}$, Eq. (2) has been put on a firmer footing using the nonlinear line charge mode ${ }^{200}$ for generic smooth emitters. The result has been verified numerically for various shapes, anode distances and even in the presence of other emitters. It was found that within about 2 nanometers from the surface of an emitter, the potential due to the applied external electric field (as determined using COMSOL) is well approximated by

$$
V_{\text {ext }}(s)=-q E_{l} s\left[1-\frac{s}{R_{2}}+\frac{4}{3}\left(\frac{s}{R_{2}}\right)^{2}\right] .
$$

At $R_{a}=5 \mathrm{~nm}$ and $E_{l}=3 \mathrm{~V} / \mathrm{nm}$, the tunneling region is well within $2 \mathrm{~nm}$, while at higher fields, the width decreases sharply (see for instance Fig. 7 of Ref. [19]).

The potential energy barrier seen by an electron can thus be expressed as

$$
V_{T}(s)=\phi+V_{\text {ext }}(s)-\frac{B}{s\left(1+\frac{s}{2 R_{2}}\right)}
$$

where the curvature corrected image potential under a locally spherical approximation has been used with $B=$ $q^{2} /\left(16 \pi \epsilon_{0}\right)$. At $T=0$, the free electrons have energy

\footnotetext{
a) Electronic mail: dbiswas@barc.gov.in
} 
$-\mathcal{E}_{F}<\mathcal{E} \leq 0$. Hereafter, we shall drop the subscript in $R_{2}$ and simply using the notation $R$.

A first step towards a curvature-corrected current density applicable at the apex of an axially symmetric emit-

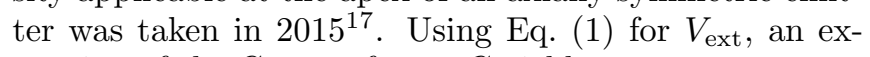
pansion of the Gamow factor $G$ yields

$$
G=g \int_{s_{1}}^{s_{2}} \sqrt{V_{T}(s)-\mathcal{E}} d s=G_{0}+x G_{1}+\mathcal{O}\left(x^{2}\right)
$$

where $g=\sqrt{8 m} / \hbar \simeq 10.246(\mathrm{eV})^{-1 / 2}(\mathrm{~nm})^{-1}$ while $s_{1}, s_{2}$ are the zeroes of the integrand at $x=(\phi-\mathcal{E}) /\left(q E_{l} R\right)=0$. Since the smallness parameter $x$ tends to zero for large $R, G_{0}$ represents the Gamow factor in the planar limit $R \rightarrow \infty$ or $x \rightarrow 0$, and $G_{1}=(\partial G / \partial x)_{\mid x=0}$ is the first curvature dependent correction. This leads in turn to a curvature corrected current density applicable at the emitter apex for small values of $x$.

TABLE I. Specification of symbols

\begin{tabular}{|l|l|}
\hline$\phi$ & Work function \\
\hline$q$ & Magnitude of electron charge \\
\hline $\mathcal{E}_{F}$ & Fermi energy \\
\hline$R_{a}$ & Apex radius of curvature \\
\hline$R_{2}$ & Second principle radius of curvature \\
\hline$E_{l}$ & Local electric field on emitter surface \\
\hline$E_{0}$ & Macroscopic electric field \\
\hline$\gamma=E_{l} / E_{0}$ & Local field enhancement factor \\
\hline$G$ & Gamow factor in WKB approximation \\
\hline$G_{\text {Exact }}$ & $G$ evaluated numerically \\
\hline$G^{(3)}$ & Approximate expression for $G$ \\
\hline$J_{\text {WKB }}^{\text {Exact }}$ & Current density using $G_{\text {Exact }}$ \\
\hline$J_{\text {WKB }}^{(3)}$ & Current density using $G^{(3)}$ \\
\hline$J_{\text {Exact }}$ & Current density obtained numerically \\
\hline$\lambda_{P}^{\text {fit }}$ & $J_{\text {WKB }}^{(3)} / J_{\text {Exact }} \approx 0.3400 \mathcal{E}_{F} / \phi+0.3614$ \\
\hline$J_{\text {Corr }}^{(3)}$ & $J_{\text {WKB }}^{(3)} \lambda_{P}^{\text {fit }}$ \\
\hline$J_{\text {WKB }}^{(0)}$ & Murphy-Good current density \\
\hline & \\
\hline
\end{tabular}

Our first aim here is to use Eq. (3) in the tunneling potential $V_{T}$ of Eq. (4) and determine a reasonably accurate expression (referred to as $G^{(3)}$ subsequently) which approximates $G$ across a wide range of commonly encountered emitter paramaters such as the workfunction $\phi$, the Fermi energy $\mathcal{E}_{F}$, the local field $E_{l}$ and the radius of curvature $R$. This is achieved in section II] In section III] the current density obtained using this transmission coefficient is compared with the exact-WKB current density ( $G$ obtained numerically) as well as the exact current density (transmission coefficient obtained by solving the Schrödinger equation numerically). Finally, we use the approximate expression $G^{(3)}$ to arrive at a curvature corrected analytical expression for the current density $J_{\mathrm{CCMG}}$ in section IV. Our results are summarized in the concluding section. A list of some of the symbols used are summarized in Table 1 .

\section{AN APPROXIMATE EXPRESSION FOR THE GAMOW FACTOR}

The Gamow factor can be expressed in terms of suitable normalized variables ${ }^{17} x, y$, and $\xi$. With $\varphi=\phi-\mathcal{E}$, they are expressed as $x=\varphi /\left(q E_{l} R\right), y=2 \sqrt{B E_{l}} / \varphi$ and $\xi=\left(q E_{l} / \varphi\right) z$. Using Eq. (3) in the the tunneling potential of Eq. (4), the Gamow factor can thus be written as

$$
G(x, y)=\frac{2}{3} g \frac{\varphi^{3 / 2}}{q E_{l}} \Xi(x, y)
$$

where

$$
\begin{gathered}
\Xi(x, y)=\frac{3}{2} \int_{\xi_{1}}^{\xi_{2}} d \xi\left(1-\xi+x \xi^{2}-\frac{4}{3} x^{2} \xi^{3}-\right. \\
\left.\frac{y^{2} / 4}{\xi+x \xi^{2} / 2}\right)^{1 / 2}
\end{gathered}
$$

where $\xi_{1}$ and $\xi_{2}$ are the real roots of

$$
1-\xi+x \xi^{2}-\frac{4}{3} x^{2} \xi^{3}-\frac{y^{2} / 4}{\xi+x \xi^{2} / 2}
$$

in the region $\xi>0$. Note that $x=0$ gives us the planar result $\Xi(0, y)=v(y) \approx 1-y^{2}+\left(y^{2} / 3\right) \ln (y)$.

For curved emitters (i.e. $x \neq 0$ ), a possible way forward is to use a Taylor expansion of $\Xi(x, y)$

$$
\Xi(x, y)=\Xi(0, y)+\sum_{k=1}^{k=N} \frac{x^{k}}{k}\left(\frac{\partial^{k} \Xi(x, y)}{\partial x^{k}}\right)_{x=0}+\mathcal{O}\left(x^{4}\right)
$$

and hope to achieve convergence in the desired domain of $x$ and $y$, for instance by restricting to $N=3$. Since expressions for each of the partial derivatives have to be evaluated numerically, it is less cumbersome to evaluate $\Xi(x, y)-\Xi(x, 0)$ directly instead of a Taylor expansion. It is this approach that we shall adopt here.

We thus choose a large number of values of $y$ in the interval $(0,1)$. For each of these $y_{i}$, we evaluate $\Xi\left(x, y_{i}\right)-$ $\Xi\left(0, y_{i}\right)$ and fit a function

$$
\Xi\left(x, y_{i}\right)-\Xi\left(0, y_{i}\right) \approx x a_{1}^{(i)}+x^{2} a_{2}^{(i)}+x^{3} a_{3}^{(i)}
$$

and determine the coefficients $a_{k}^{(i)}, k=1,2,3$. This is then repeated for all values of $y$ chosen. A typical fit $\left(y_{i}=0.25\right)$ is shown in Fig. 1 . 


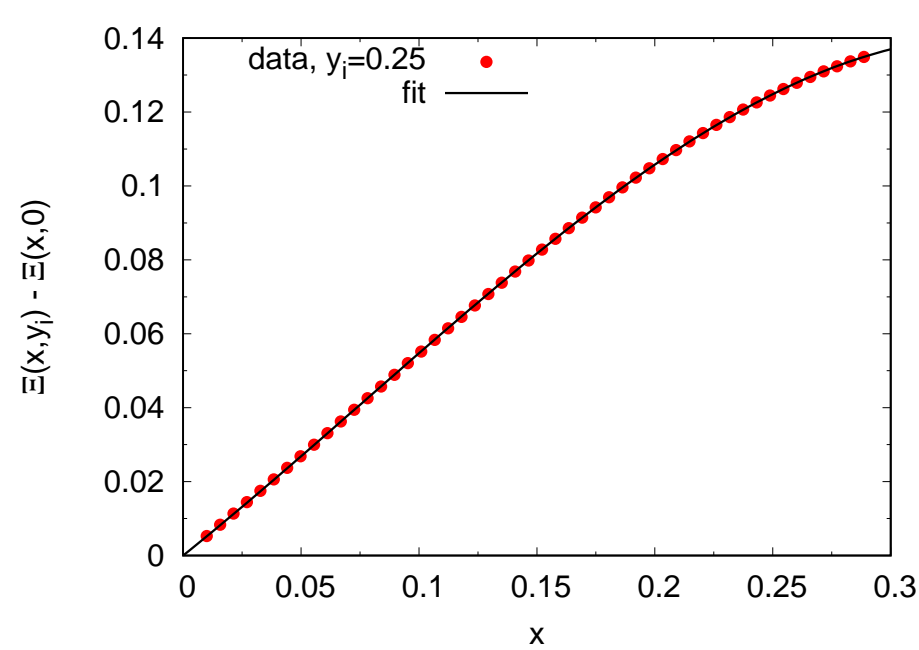

FIG. 1. Variation of $\Xi\left(x, y_{i}\right)-\Xi(x, 0)$ for $y_{i}=0.25$ and the fitted function.

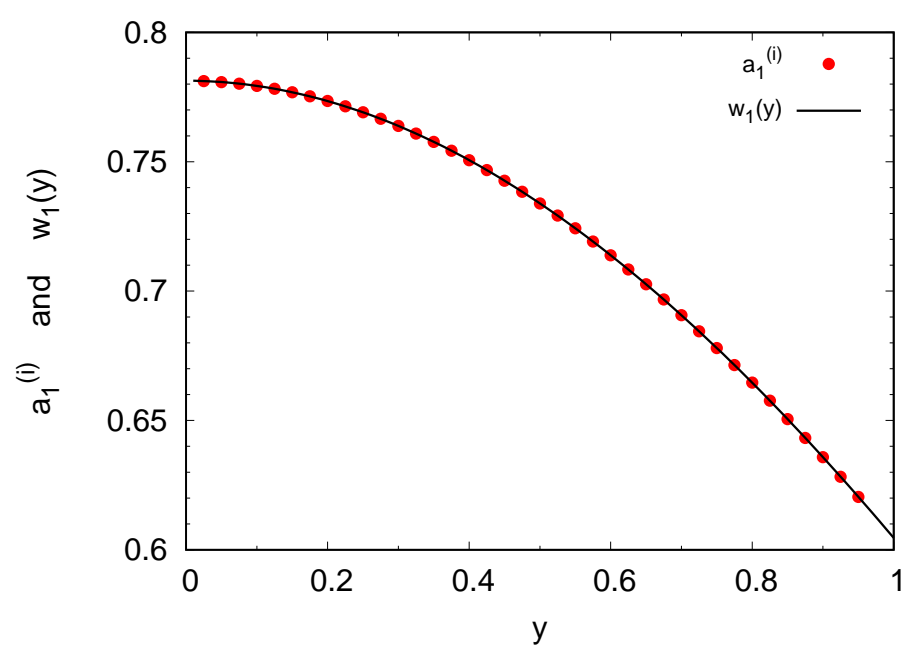

FIG. 2. Variation of the coefficients $\left\{a_{1}^{(i)}\right\}$ and the fitted function $w_{1}(y)$.

Each of three sets of coefficients $\left\{a_{1}^{(i)}\right\},\left\{a_{2}^{(i)}\right\}$ and $\left\{a_{3}^{(i)}\right\}$ can be used to express their variation with $y$ using a fitting function in line with the form used for $v(y)$. We choose the functions $w_{k}=c_{0}^{(k)}+c_{1}^{(k)} y^{2}+c_{2}^{(k)} y^{4}+$ $c_{3}^{(k)} y^{2} \ln \left(y^{2}\right)$ and determine the coefficients $c_{j}^{(k)}, j=$ $0,1,2,3$ by fitting to $\left\{a_{k}^{(i)}\right\}$. The data $\left\{a_{k}^{(i)}\right\}$ and the fitted functions $w_{k}$ are shown in Figs. 2-4.

The fits in each of the three cases is excellent. Note that the quartic term was required to get a better fit over the entire range of $y$ values. Also, note that $\left\{a_{1}^{i}\right\}$ are close in value to the function $w(y)$ of Ref. [17 but not identical. This is due to the fact that $\Xi\left(x, y_{i}\right)$ is not restricted to very small values of $x$ while evaluating the coefficients $\left\{a_{1}^{i}\right\}$. For $x \in[0,0.02]$, the value of $a_{1}$ for $y=0.025$ is about $0.805 \approx 4 / 5$ as in Ref. [17.

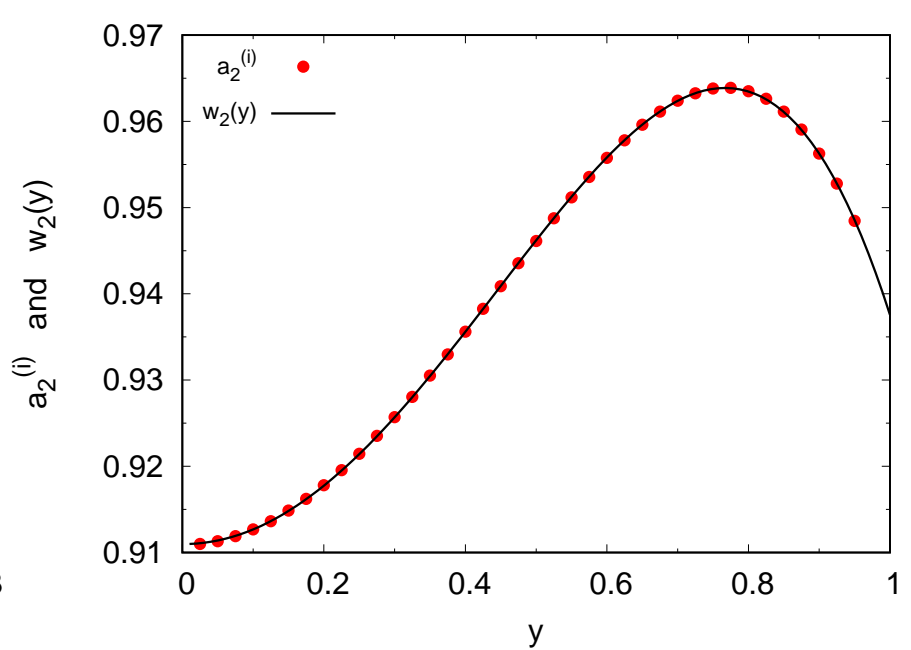

FIG. 3. Variation of the coefficients $\left\{a_{2}^{(i)}\right\}$ and the fitted function $w_{2}(y)$.

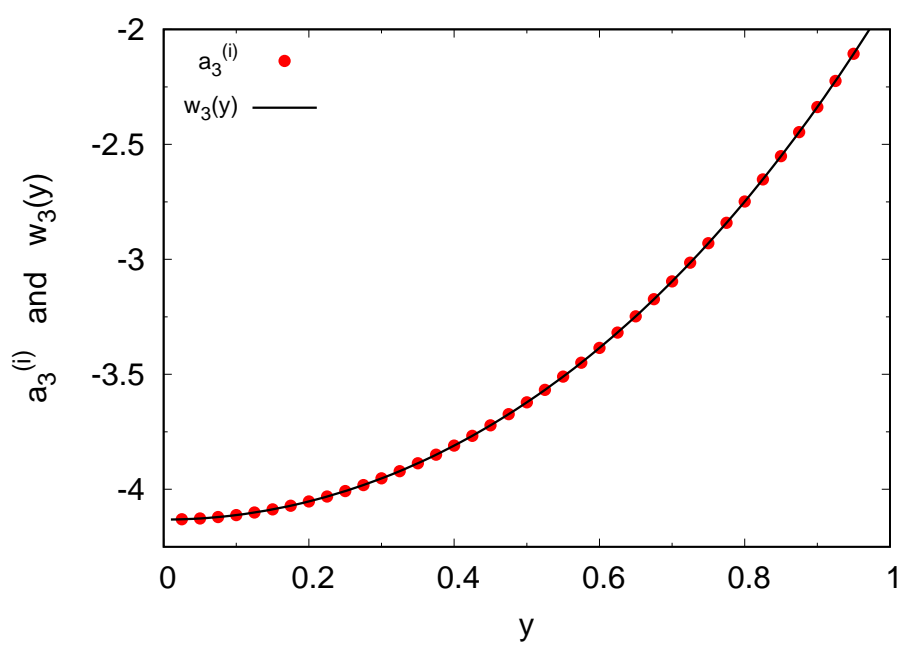

FIG. 4. Variation of the coefficients $\left\{a_{3}^{(i)}\right\}$ and the fitted function $w_{3}(y)$.

The fitting coefficients $c_{j}^{(k)}, j=0,1,2,3$ can be suitably approximated so that as

$$
\begin{aligned}
& w_{1}(y)=\frac{10}{13}-\frac{2}{11} y^{2}+\frac{1}{80} y^{4}+\frac{1}{400} y^{2} \ln \left(y^{2}\right) \\
& w_{2}(y)=\frac{10}{11}+\frac{2}{11} y^{2}-\frac{1}{6} y^{4}+\frac{1}{400} y^{2} \ln \left(y^{2}\right) \\
& w_{3}(y)=-\frac{41}{10}+\frac{39}{20} y^{2}+\frac{1}{3} y^{4}-\frac{1}{300} y^{2} \ln \left(y^{2}\right) .
\end{aligned}
$$

Together with $v(y)$,

$G^{(3)}(x, y)=\frac{2}{3} g \frac{\varphi^{3 / 2}}{q E_{l}}\left[v(y)+x w_{1}(y)+x^{2} w_{2}(y)+x^{3} w_{3}(y)\right]$ 
and the transmission coefficient $T(\mathcal{E})$ can be approximated as

$$
T(\mathcal{E}) \simeq e^{-G^{(3)}(x, y)}
$$

Note that the exact tranmission coefficient evaluated by solving the Schrödinger equation numerically differs from Eq. (14) by a factor $P$ which depends on $x$ and $y$.

\section{COMPARISON WITH EXACT RESULTS}

Rather than directly comparing the transmission coefficients evaluated using Eq. (11)-(14) with an exact numerical scheme such as the transfer matrix method ${ }^{21}$, we shall compare instead the current densities evaluated using these methods.

The current density within the free electron model can be evaluated to determine the accuracy of the results. At zero temperature,

$$
J=\frac{2 m q}{(2 \pi)^{2} \hbar^{3}} \int_{0}^{\mathcal{E}_{F}} T\left(\mathcal{E}^{\prime}\right) \mathcal{E}^{\prime} d \mathcal{E}^{\prime}
$$

where $T(\mathcal{E})$ is the transmission coefficient at electron energy $\mathcal{E}$ measured with respect to the top of the conduction band, while $\mathcal{E}_{F}$ is the Fermi energy and $m$ the mass of the electron.

The efficacy of Eq. (11)-14 for evaluating the transmission coefficient $T(\mathcal{E})$ can be judged in several ways. In the first instance $J$ can be evaluated using Eq. (15) and Eq. 11)- 14 . We shall refer to this as $J_{\mathrm{WKB}}^{(3)}$ to denote that $T(\mathcal{E})$ is evaluated using WKB and incorporates terms upto $x^{3}$. The current density can also be evaluated using Eq. 15 with $T(\mathcal{E})=e^{-G_{\text {Exact }}(x, y)}$ where $G_{\text {Exact }}(x, y)$ is determined by numerically integrating $\Xi(x, y)$ from $\xi_{1}$ to $\xi_{2}$. We shall refer to this as $J_{\text {WKB }}^{\text {Exact }}$. Finally, $J$ can also be evaluated by numerically determining the transmission coefficient $T(E)$ exactly using the transfer matrix or solving the Schrödinger equation numerically 21 , and using it in Eq. 15 . We shall refer to this last evaluation of the current density as $J_{\text {Exact }}$.

Each of the three current densities, $J_{\mathrm{WKB}}^{(3)}, J_{\mathrm{WKB}}^{\text {Exact }}$ and $J_{\text {Exact }}$ have been evaluated at 10,000 points respectively corresponding to 10 points each for $\mathcal{E}_{F} \in[5,10] \mathrm{eV}$, $\phi \in[3,5.5] \mathrm{eV}, E_{l} \in[3,10] \mathrm{V} / \mathrm{nm}$ and $R \in[5,40] \mathrm{nm}$. We choose to display these by plotting the errors as a function of $E_{l} R /\left(\mathcal{E}_{F}+\phi\right)$.

Fig. 5 shows the scatter plot of relative error $100 \times$ $\left|J_{\mathrm{WKB}}^{(3)}-J_{\mathrm{WKB}}^{\text {Exact }}\right| / J_{\mathrm{WKB}}^{\text {Exact }}$. Most points have an error less than $2 \%$ while the average error is $1.56 \%$. The maximum error is below $5 \%$.

Fig. 6 shows the scatter plot of relative error $100 \times$ $\left|J_{\text {WKB }}^{(3)}-J_{\text {Exact }}\right| / J_{\text {Exact }}$. The errors can be large as seen from the scatter plot with the maximum error at around $55 \%$ while the average error is $14.66 \%$. The difference is

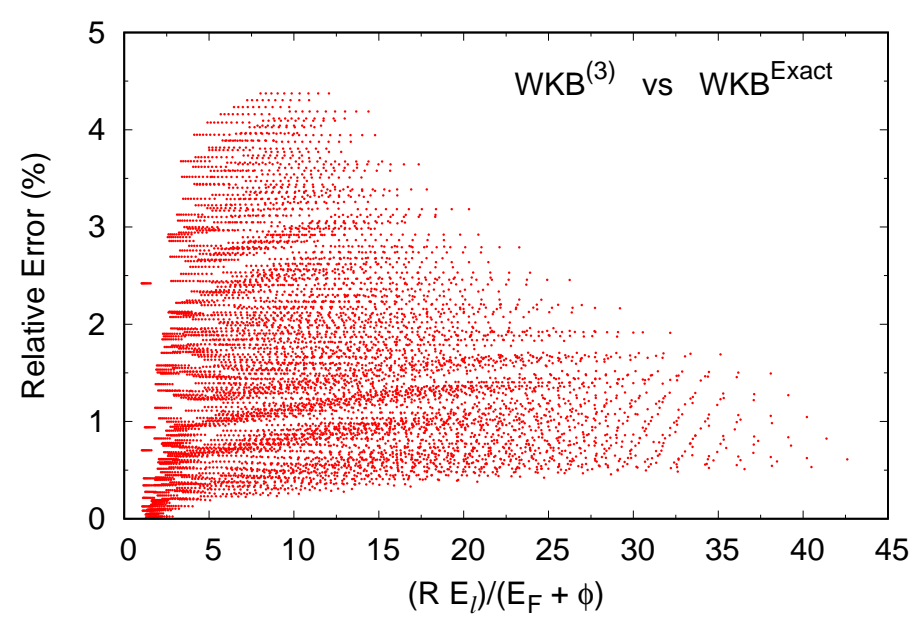

FIG. 5. A scatter plot of the relative error $100 \times \mid J_{\mathrm{WKB}}^{(3)}-$ $J_{\mathrm{WKB}}^{\text {Exact }} \mid / J_{\mathrm{WKB}}^{\text {Exact }}$.

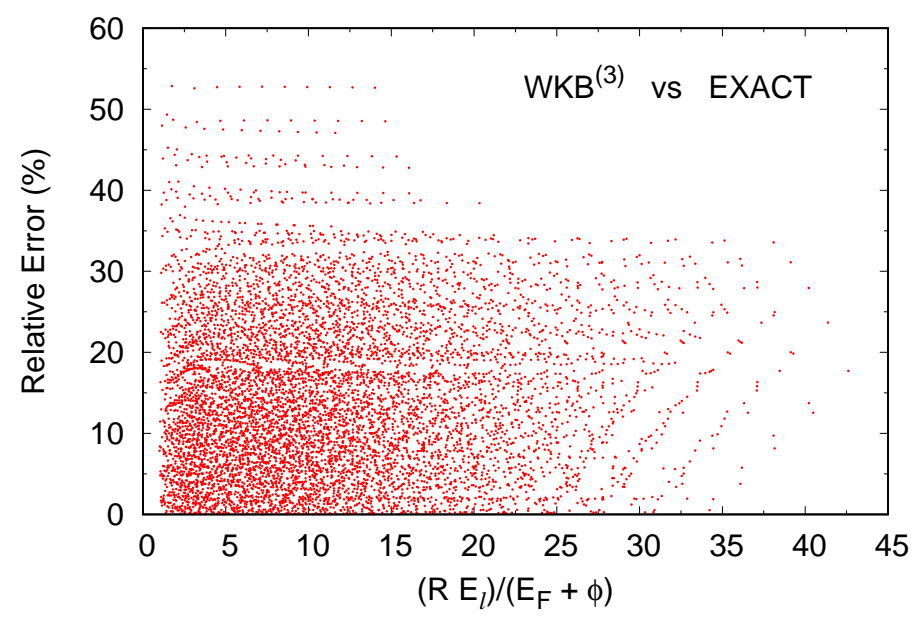

FIG. 6. A scatter plot of the relative error $100 \times \mid J_{\mathrm{WKB}}^{(3)}-$ $J_{\text {Exact }} \mid / J_{\text {Exact }}$.

due to the factor $P$ which relates the exact and WKB transmission coefficients.

In order to reduce the large difference between $J_{\mathrm{WKB}}^{(3)}$ and $J_{\text {Exact }}$, we shall study the ratio $\lambda_{P}=J_{\mathrm{WKB}}^{(3)} / J_{\text {Exact }}$. Clearly $\lambda_{P}$ depends on $\mathcal{E}_{F}, \phi, E_{l}$, and $R$. An expansion of $\lambda_{P}$ in terms of these variables along the lines of Ref. 22] can in principle be carried out. Our interest here to find a simple expression for $\lambda_{P}$ that is easy to use and reduces the average error substantially.

Fig. 7 shows $\lambda_{P}=J_{\mathrm{WKB}}^{(3)} / J_{\text {Exact }}$ plotted against $\mathcal{E}_{F} / \phi$. Clearly, the points seems to display a linear increase with $\mathcal{E}_{F} / \phi$. The best fitting straight line $\lambda_{P}^{\text {fit }}=a_{0} \mathcal{E}_{F} / \phi+b_{0}$ is also shown in figure with $a_{0} \approx 0.3400$ and $b_{0} \approx 0.3614$.

The corrected current density $J_{\mathrm{Corr}}^{(3)}=J_{\mathrm{WKB}}^{(3)} / \lambda_{P}^{\mathrm{fit}}=$ $J_{\mathrm{WKB}}^{(3)} /\left(a_{0} \mathcal{E}_{F} / \phi+b_{0}\right)$ does not eliminate the error completely but is expected to reduce it substantially. Fig. 8 is a scatter plot of the relative error $100 \times \mid J_{\text {Corr }}^{(3)}-$ 


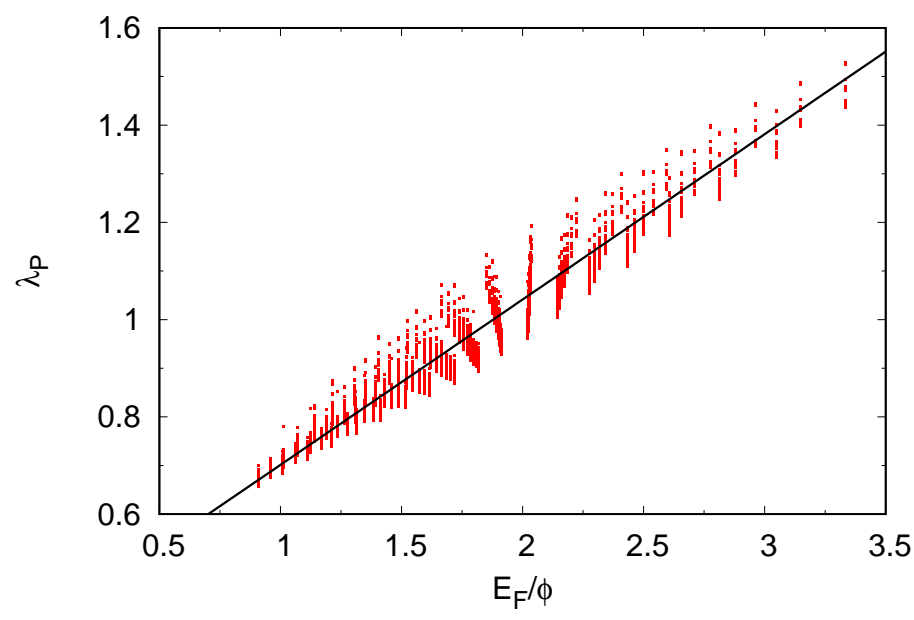

FIG. 7. The factor $\lambda_{P}=J_{\mathrm{WKB}}^{(3)} / J_{\text {Exact }}$ plotted against $\mathcal{E}_{F} / \phi$. Also shown is the best fitting straight line.

$J_{\text {Exact }} \mid / J_{\text {Exact }}$. The average error is $3.58 \%$ and the maximum error has also reduced substantially.

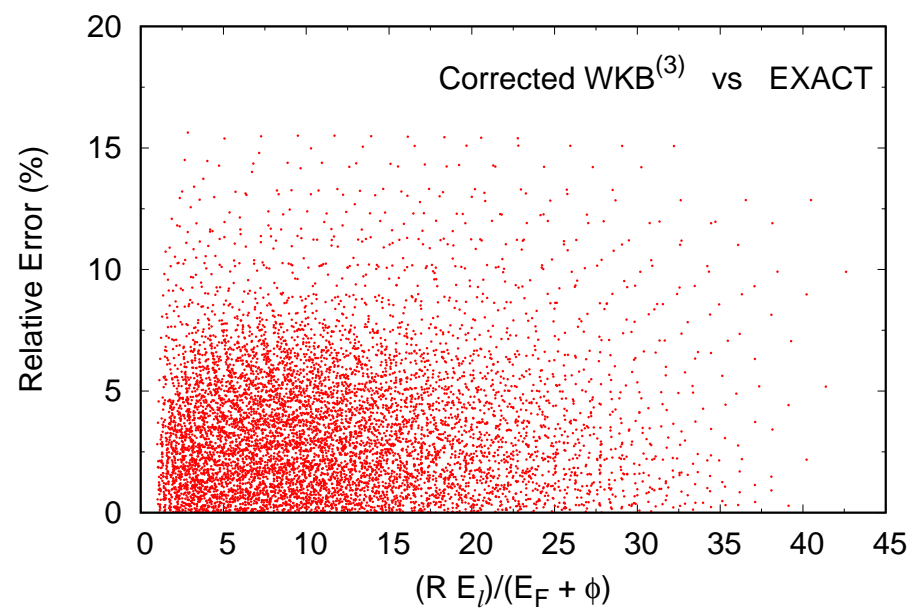

FIG. 8. A scatter plot of the relative error $100 \times \mid J_{\text {Corr }}^{(3)}-$ $J_{\text {Exact }} \mid / J_{\text {Exact }}$.

Clearly the curvature corrected WKB current density $J_{\text {WKB }}^{(3)}$ is accurate over a wide range of parameters. It can be used along with $\lambda_{P}^{\text {fit }}$ to get a reasonably accurate current density $J_{\text {Corr }}^{(3)}$ which compares well with the exact current density.

\section{CORRECTIONS TO THE CURRENT DENSITY}

The curvature-corrected zero-temperature current density can be evaluated using Eq. (15), Eq. (14) and Eq. 13. The prefactor $\lambda_{P}^{\text {fit }}$ can be optionally used to get a more accurate expression.

\section{A. An analytical expression}

Note that the dominant contribution to the integral in Eq. 15 is close to the Fermi level $\left(\mathcal{E}^{\prime}=0\right)$ since $T\left(\mathcal{E}^{\prime}\right)$ decreases sharply with $\mathcal{E}^{\prime}$. A Taylor expansion of $G$ at $\mathcal{E}=0$ is therefore used ${ }^{4}$. Thus,

$$
G(\mathcal{E})=G(0)+\mathcal{E}\left(\frac{\partial G}{\partial \mathcal{E}}\right)_{\mathcal{E}=0}+\mathcal{O}\left(\mathcal{E}^{2}\right)
$$

An expression for $\left(\frac{\partial G}{\partial \mathcal{E}}\right)_{\left.\right|_{\mathcal{E}=0}}$ can be easily obtained by noting that $\left(\frac{\partial x}{\partial \mathcal{E}}\right)_{\left.\right|_{\mathcal{E}=0}}=-x_{F} / \phi$ and $\left(\frac{\partial y}{\partial \mathcal{E}}\right)_{\left.\right|_{\mathcal{E}=0}}=y_{F} / \phi$ where $x_{F}=\phi /\left(q E_{l} R\right)$ and $y_{F}=2 \sqrt{B E_{l}} / \phi$. Thus

$$
\begin{aligned}
\left(\frac{\partial G}{\partial \mathcal{E}}\right)_{\mathcal{E}=0}= & \frac{1}{d_{F}}\left[\left\{v\left(y_{F}\right)-\frac{2}{3} y_{F}\left(\frac{d v}{d y}\right)_{y=y_{F}}\right\}+\right. \\
& x_{F}\left\{\frac{5}{3} w_{1}\left(y_{F}\right)-\frac{2}{3} y_{F}\left(\frac{d w_{1}}{d y}\right)_{y=y_{F}}\right\}+ \\
& x_{F}^{2}\left\{\frac{7}{3} w_{2}\left(y_{F}\right)-\frac{2}{3} y_{F}\left(\frac{d w_{2}}{d y}\right)_{y=y_{F}}\right\}+ \\
x_{F}^{3} & \left.\left\{3 w_{3}\left(y_{F}\right)-\frac{2}{3} y_{F}\left(\frac{d w_{3}}{d y}\right)_{y=y_{F}}\right\}\right]
\end{aligned}
$$

where $d_{F}^{-1}=g \phi^{1 / 2} /\left(q E_{l}\right)$. This can be further simplified and expressed as

$$
\left(\frac{\partial G}{\partial \mathcal{E}}\right)_{\mathcal{E}=0}=-\frac{t\left(y_{F}\right)+x_{F} \psi_{1}\left(y_{F}\right)+x_{F}^{2} \psi_{2}\left(y_{F}\right)+x_{F}^{3} \psi_{3}\left(y_{F}\right)}{d_{F}}
$$

where on using $y_{F}^{2}=f$,

$$
\begin{aligned}
t & =1+\frac{f}{9}-\frac{1}{18} f \ln (f) \\
\psi_{1} & =\frac{25}{13}-\frac{237}{1100} f-\frac{1}{480} f^{2}-\frac{7}{2400} f \ln (f) \\
\psi_{2} & =\frac{70}{33}+\frac{589}{3300} f+\frac{1}{18} f^{2}+\frac{1}{400} f \ln (f) \\
\psi_{3} & =-\frac{123}{10}+\frac{2929}{900} f+\frac{1}{9} f^{2}-\frac{1}{180} f \ln (f) .
\end{aligned}
$$

Thus, finally using Eqns. (14)-(16), the current density can be finally expressed as

$$
J=\frac{2 m q}{(2 \pi)^{2} \hbar^{3}} \frac{d_{F}^{2}}{t_{c}^{2}} e^{-v_{c} B_{F N} \phi^{3 / 2} / E_{l}}
$$

where $v_{c}$ and $t_{c}$ are the correction factors due to emitter curvature and image potential. They are 


$$
\begin{aligned}
v_{c}(f)= & \left(1-f+\frac{1}{6} f \ln (f)\right)+ \\
& x_{F}\left(\frac{10}{13}-\frac{2}{11} f+\frac{1}{80} f^{2}+\frac{1}{400} f \ln (f)\right)+ \\
& x_{F}^{2}\left(\frac{10}{11}+\frac{2}{11} f-\frac{1}{6} f^{2}+\frac{1}{400} f \ln (f)\right)+ \\
& x_{F}^{3}\left(-\frac{41}{10}+\frac{39}{20} f+\frac{1}{3} f^{2}-\frac{1}{300} f \ln (f)\right)
\end{aligned}
$$

and

$$
\begin{aligned}
t_{c}(f) & =\left(1+\frac{f}{9}-\frac{1}{18} f \ln (f)\right)+ \\
x_{F} & \left(\frac{25}{13}-\frac{237}{1100} f-\frac{1}{480} f^{2}-\frac{7}{2400} f \ln (f)\right)+ \\
x_{F}^{2} & \left(\frac{70}{33}+\frac{589}{3300} f+\frac{1}{18} f^{2}+\frac{1}{400} f \ln (f)\right)+ \\
x_{F}^{3} & \left(-\frac{123}{10}+\frac{2929}{900} f+\frac{1}{9} f^{2}-\frac{1}{180} f \ln (f)\right) .
\end{aligned}
$$

The final expression for the curvature-correctedMurphy-Good (CCMG) current density in terms of the conventional Fowler-Nordheim constant $A_{\mathrm{FN}}$ and $B_{\mathrm{FN}}$ takes the form

$$
J_{\mathrm{CCMG}}=\frac{A_{F N}}{\phi} \frac{E_{l}^{2}}{t_{c}^{2}} \exp \left(-v_{c} B_{F N} \phi^{3 / 2} / E_{l}\right)
$$

where $E_{l}$ refers to the local electric field on the emitter surface, $A_{\mathrm{FN}} \simeq 1.541434 \mu \mathrm{A} \mathrm{eV} \mathrm{V}{ }^{-2}, B_{\mathrm{FN}} \simeq$ $6.830890 \mathrm{eV}^{-3 / 2} \mathrm{~V} \mathrm{~nm}^{-1}, f \simeq 1.439965 E_{l} / \phi^{2}$ and $x_{F}=\phi /\left(q E_{l} R\right)$.

Equations 24)-26 provide a direct means of evaluating the local current density instead of numerically evaluating the integral in Eq. 15.

\section{B. Comparison with exact-WKB result}

It may be noted that the curvature-corrected MurphyGood formula of Eq. (26) is an approximation of the integral in Eq. 15 evaluated using Eq. (14), and is not necessarily accurate. At smaller values of the local field, electrons close to the Fermi level are expected to contribute and hence Eq. (16) is likely to be adequate. With an increase in local field strength, electrons further away from the Fermi level can tunnel through due to the decrease in height and width of the barrier. The truncation of the series in Eq. (16) may thus lead to errors at higher applied fields. Such a problem in fact exists even in the commonly used Murphy-Good expression for current density as we shall see. The energy-integration error is expected to assume significance in $J_{\mathrm{CCMG}}$ for large $R$ while errors at small $R$ may be due to curvature effects.
In the following, we shall test how well $J_{\mathrm{CCMG}}$ approximates $J_{\mathrm{WKB}}^{\text {Exact }}$ across a range of radius of curvature given that there are two levels of approximation involved in going from $J_{\mathrm{WKB}}^{\text {Exact }}$ to $J_{\mathrm{CCMG}}$ given by Eq. 26). The first of these is the use of $G^{(3)}$ instead of $G_{\text {Exact }}$ while the second involves the energy integration mentioned above. It is the combined effect of the two that will manifest as the relative error.

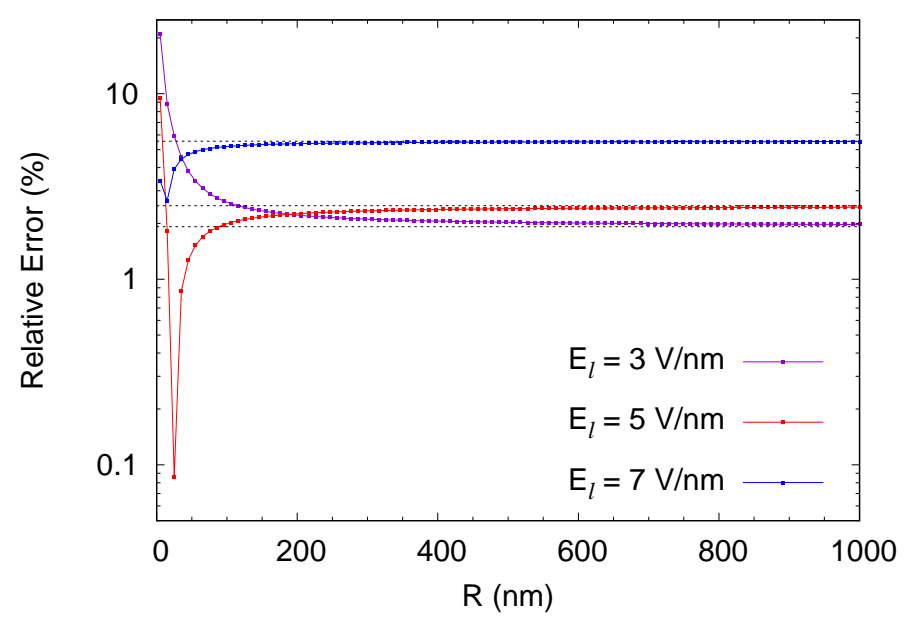

FIG. 9. Variation in relative error $100 \times \mid J_{\mathrm{CCMG}}-$ $J_{\mathrm{WKB}}^{\text {Exact }} \mid / J_{\mathrm{WKB}}^{\text {Exact }}$ with radius $R$. Here $\phi=4.5 \mathrm{eV}$ and $\mathcal{E}_{F}=$ $8.5 \mathrm{eV}$.

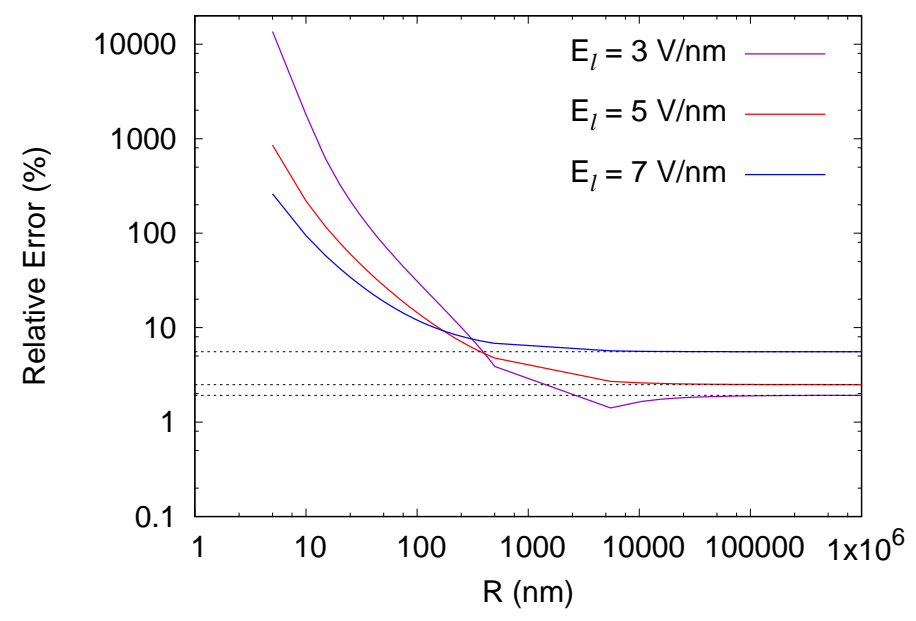

FIG. 10. Variation in relative error $100 \times \mid J_{\mathrm{WKB}}^{(0)}-$ $J_{\mathrm{WKB}}^{\text {Exact }} \mid / J_{\mathrm{WKB}}^{\text {Exact }}$ with radius $R$. Here $\phi=4.5 \mathrm{eV}$ and $\mathcal{E}_{F}=$ $8.5 \mathrm{eV}$.

For simplicity, we consider a fixed value $\phi=4.5 \mathrm{eV}$ and $\mathcal{E}_{F}=8.5 \mathrm{eV}$ but three different values of the local field, $E_{l}$. The radius $R$ is varied and the relative error $100 \times\left|J_{\mathrm{CCMG}}-J_{\mathrm{WKB}}^{\mathrm{Exact}}\right| / J_{\mathrm{WKB}}^{\mathrm{Exact}}$ is recorded. The results are shown in Fig. 9. The dashed lines mark the error in $J_{\mathrm{CCMG}}$ at large $R$ and their values coincide with the error in planar Murphy-Good result (i.e. using $x_{F}=0$ in Eq. (26)) for different values of the local field. As expected, the asymptotic error ( $R$ large) is small at $E_{l}=$ $3 \mathrm{~V} / \mathrm{nm}$ but increases with $E_{l}$. On the other hand, at 
smaller radius of curvature $(R<50 \mathrm{~nm})$, the error is small at higher values of the local field ( $x_{F}$ small) but increases at lower values of local field. This is again expected since $x_{F}$ becomes larger as $E_{l}$ decreases and $G^{(3)}$ does not approximate $G_{\text {Exact }}$ as well. For $R>50 \mathrm{~nm}$, the error due to energy integration seems to dominate and appears to be the limiting factor.

It is also instructive to see how well the curvatureuncorrected current density $J_{\mathrm{WKB}}^{(0)}$ fares in dealing with the curvature-dependent tunneling potential. Fig. 10 shows the relative error $100 \times\left|J_{\mathrm{WKB}}^{(0)}-J_{\mathrm{WKB}}^{\text {Exact }}\right| / J_{\mathrm{WKB}}^{\text {Exact }}$ where $J_{\mathrm{WKB}}^{(0)}$ is evaluated by setting $x_{F}=0$ in Eq. 26, while $J_{\mathrm{WKB}}^{\mathrm{Exact}}$ is evaluated as before using the tunneling potential of Eq. (4) with the curvature corrections. The convergence to the strictly planar error limit is slow for each of the three local fields. At small $R$, the error is very high in each of three cases with the lowest field having the highest error as expected.

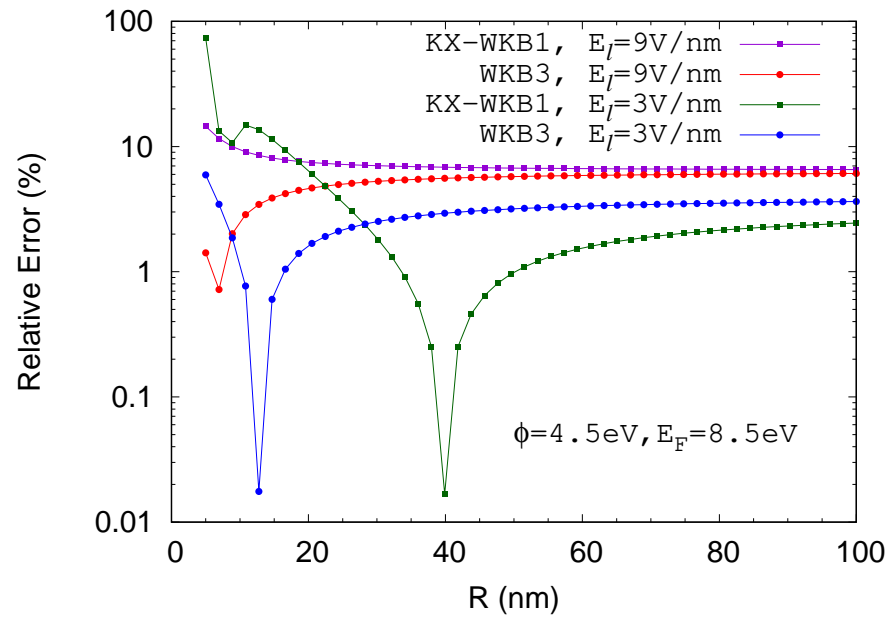

FIG. 11. Variation in relative error with radius $R$. Here $\phi=5.0 \mathrm{eV}$ and $\mathcal{E}_{F}=8.5 \mathrm{eV}$.

The first order curvature-corrected current density of Kyritsakis and Xanthaki ${ }^{17}$ (denoted by KX-WKB1) can also be compared to $J_{\mathrm{WKB}}^{\text {Exact }}$ since a Taylor expansion of the Gamow factor at $x=0$ using the tunneling potential of Eq. (4) gives identical result. The result is expected to be good at larger local fields and radius of curvature. Figure 11 shows a comparison of the relative errors for KX-WKB1 and $J_{\mathrm{CCMG}}$ (denoted by WKB3) with respect to $J_{\mathrm{WKB}}^{\mathrm{Exact}}$ at 2 different local fields. For KX-WKB1, $v_{c}=$ $\left(1-f+\frac{f}{6} \ln f\right)+x_{F}\left(\frac{4}{5}-\frac{7}{40} f+\frac{1}{200} \ln f\right)$ while $t_{c}=(1+$ $\left.\frac{f}{9}-\frac{f}{18} \ln f\right)+x_{F}\left(\frac{4}{3}-\frac{f}{15}-\frac{f \ln f}{1200}\right)$. Note again that energyintegration errors begin to dominate in both cases with an increase in radius of curvature while at smaller values of $R$, this error is somewhat suppressed since the barrier is wider and Eq. (16) may be adequate. It is however clear that for small $R$ and $E_{l}$, there is a clear advantage in using $J_{\mathrm{CCMG}}$.

\section{v. CONCLUSIONS}

We have considered higher order curvature corrections to the current density for the near-universal tunneling potential of Eq. (4), which contains quadratic and cubic curvature-dependent terms in the external potential.

This has been achieved by first finding a suitable expression which accurately represents the exact Gamow factor for a range of local field, radius of curvature, Fermi energy, work function and electron energy. This was used to compute the current density by integrating over the energy states numerically and compared with the exact-WKB current density. The errors were found to be small for a range of parameters thereby validating the expression for the Gamow factor presented in this paper. We then proceeded to determine an expression for the curvature-corrected current density $J_{\mathrm{CCMG}}$, following the standard procedure of expanding the transmission coefficient at the Fermi level and carrying out the energy integration in Eq. (15). The errors relative to the exactWKB current density was again found to be reasonably small. In comparison, the standard planar Murphy-Good result was found to have very large error at smaller radius of curvature and local fields. For larger radius of curvature $(R>50 \mathrm{~nm})$, the error in the curvature-corrected current density $J_{\mathrm{CCMG}}$ appears to be dominated by the errors in the energy integration.

We also studied the problem of discrepancy between the 'exact' and exact-WKB current densities and discovered a pattern in their ratio when plotted against $\mathcal{E}_{F} / \phi$. This enabled us to suggest a simple prefactor $\lambda_{P}^{\text {fit }}$ to correct the exact-WKB current density. The average error over a range of parameters was found to be about $3.58 \%$. Keeping in mind the underlying uncertainties in modeling the geometric and material properties of emitters and their effect on the field emission current, errors below $10 \%$ are clearly acceptable.

Finally, the curvature-corrected expression for current density $J_{\mathrm{CCMG}}$ can be combined with the cosine law $24 \mid 25$ of variation of the local field on the surface of generic parabolic emitter tips, for determining an approximate expression for the total field emission current and distributions of emitted particles with respect to launch angle, total and normal energy along the lines of Ref. 26] where an emitter with large apex radius of curvature was considered.

\section{ACKNOWLEDGEMENTS}

The authors acknowledge useful discussions with Dr. Raghwendra Kumar.

Data Availability: The computational data that supports the findings of this study are available within the article. 


\section{REFERENCES}

${ }^{1}$ R. H. Fowler and L. Nordheim, Proc. R. Soc. A 119, 173 (1928).

${ }^{2}$ L. Nordheim, Proc. R. Soc. A 121, 626 (1928).

${ }^{3}$ R. E. Burgess, H. Kroemer, J. M. Houston, Phys. Rev. 90, 515 (1953).

${ }^{4}$ E. L. Murphy and R. H. Good, Phys. Rev. 102, 1464 (1956).

${ }^{5}$ K. L. Jensen, J. Vac. Sci. Technol. B 21, 1528 (2003).

${ }^{6}$ R. G. Forbes, App. Phys. Lett. 89, 113122 (2006).

${ }^{7} \mathrm{~K}$. L. Jensen, Introduction to the physics of electron emission, Chichester, U.K., Wiley, 2018.

${ }^{8}$ R. G. Forbes and J. H. B. Deane, Proc. R. Soc. A 463, 2907 (2007).

${ }^{9}$ J. H. B. Deane and R. G. Forbes, J. Phys. A: Math. Theor. 41, 395301 (2008).

${ }^{10}$ K. L. Jensen, J. Appl. Phys. 126, 065302 (2019).

${ }^{11}$ D. Biswas and R. Ramachandran, J. Vac. Sci. Technol. B 37, 021801 (2019).

${ }^{12}$ D. Biswas and R. Kumar, J. Vac. Sci. Technol. B 37, 040603 (2019).

${ }^{13}$ C. J. Edgcombe, and U. Valdrè, Philosophical Magazine B 82, 987 (2002).
${ }^{14}$ R. G. Forbes, C. J. Edgcombe, and U. Valdrè, Ultramicroscopy 95, 57 (2003)

${ }^{15}$ D. Biswas, Phys. Plasmas 25, 043113 (2018).

${ }^{16}$ D. Biswas and R. Rudra, Physics of Plasmas 25, 083105 (2018).

${ }^{17}$ A. Kyritsakis and J. P. Xanthakis, Proc. R. Soc. London, A471, $20140811(2015)$

${ }^{18}$ D. Biswas, R. Ramachandran and G. Singh, Physics of Plasmas, 25, 013113 (2018).

${ }^{19} \mathrm{R}$. Ramachandran and D. Biswas, 'Universality in the tunneling potential for curved field emitters - a line charge model approach', preprint (2021) https://arxiv.org/abs/2103.01567

${ }^{20}$ D. Biswas, G. Singh and R. Kumar, J. Appl. Phys. 120, 124307 (2016).

${ }^{21}$ D. Biswas and V. Kumar, Phys. Rev. E 90, 013301 (2014).

${ }^{22}$ A. Mayer, J. Vac. Sci. Technol. B 29, 021803 (2011).

${ }^{23}$ In a Taylor expansion of the Gamow factor at $x=0$, the first $x$ dependent term is the same as in [17] even for the full tunneling potential which includes $\frac{4}{3} q E_{l} \frac{s^{3}}{R^{2}}$.

${ }^{24}$ D. Biswas, G. Singh, S. G. Sarkar and R. Kumar, Ultramicroscopy 185, 1 (2018).

${ }^{25}$ D. Biswas, G. Singh and R. Ramachandran, Physica E 109, 179 (2019).

${ }^{26}$ D. Biswas, Physics of Plasmas 25, 043105 (2018). 